\title{
A Quick Wideband Spectrum Sensing Algorithm Based On Analog Filter*
}

\author{
Ningning Hou \\ School of Information and Communication Engineering \\ Beijing University of Posts and Telecommunications, \\ Beijing, P. R. China \\ Email: hningning_bupt@163.com
}

\author{
Gang Xie \\ School of Information and Communication Engineering \\ Beijing University of Posts and Telecommunications, \\ Beijing, P. R. China \\ Email: xiegang@bupt.edu.cn
}

\begin{abstract}
Wideband spectrum sensing is very essential in Cognitive Radio (CR) systems. In order to improve the opportunistic throughput for mobile devices, the CR must sense the signals in multiple bands or wideband quickly and efficiently. The relevant literature has discussed a lot about OFDM and compressed sensing to deal with the wideband detection of signals. In this paper, we propose a quick analog-filter based spectrum sensing algorithm, using energy detection in each sub-band. The insight idea of this algorithm is that we use exponential sense to quickly find out the sequential sub-bands which are clear in the wideband, and linear sense to find the occupied sub-bands in the sensing window step by step. Furthermore, we design a dynamic adaptive algorithm to optimize the total time of sensing. This algorithm is much faster than sequential scan and much cheaper than using a wideband radio. Meanwhile, it can reach the same asymptotic complexity as compressed while the realization is much easier. Further performance improvement can be achieved through channel bonding. It is shown that our algorithm can obtain an excellent wideband spectrum sensing performance without the sparsity information.
\end{abstract}

Keywords-Cognitive Radio, Wideband Spectrum Sensing, Quick Sense

\section{INTRODUCTION}

The Cognitive Radio (CR), since has been proposed, holds the promise of solving the current problem of frequency spectrum scarcity. Through dynamic and opportunistic spectrum access, we expect to solve the severe spectrum shortage caused by the exponential growth of mobile data. In fact, the United States is the pioneer in investigating the usage of CR and it has already taken action. In 2002, the Federal Communication Commission established the Spectrum Policy Task Force (SPTF), which published its opinion about the usage of $\mathrm{CR}$. It holds the view that the unused spectrum in the UHF TV bands is available for unlicensed broadband wireless devices [1]. The US government has set the goal of freeing up $500 \mathrm{MHz}$ of spectrum for wireless communication to win the future through expanded wireless access [2].As another leader in this field, the

*This work is supported in part by Research Innovation Fund for College Students of Beijing University of Posts and Telecommunications, NSFC Project 61531007and 61302083.
European Communication Commission (ECC) is evaluating the use of the white spaces in the 470-790 $\mathrm{MHz}$ frequency band by CR system [3]. One of the most fundamental tasks of the CR is to obtain the spectrum map by spectrum sensing. The map provides the information of the occupancy of all channels and their usage in timeline, which gives the dynamic spectrum access devices a global perspective, further to help them to select the most suitable channel at a particular instant of time and specific geographic location.

Many papers in scientific literature focus on the spectrum sensing in narrowband. The main task in this case is to decide whether there is signal or noise in this band. Several promising algorithms for signal detection in narrowband are presented in [4] and [5], including the cyclostationary feather extraction, match filter detection, energy detection, wavelet detection and covariance detection. Compared with narrowband sensing, wideband sensing is considerably more challenging because the entire sensing space is very large. Related measurements show that there are at least several hundred spectrum holes in the band between $30 \mathrm{MHz}$ and $3 \mathrm{GHz}$, each of which has the width of more than $2 \mathrm{MHz}$ [6]. However the traditional algorithm above is not suitable enough for wideband. Many papers solve the problem in wideband with the usage of bank tunable band pass filters to sense sub-bands one by one. Then, one of the traditional algorithms can be used. In paper[7], it tries to detect the signal or the existence of specific signatures by energy detection. In [8] and [9], a wavelet-based multi-resolution sensing algorithm is introduced. In [10] and [11], an eigenvalue-based algorithm is used to sense sub-bands, one sub-band at one time. Finally, authors in [12] proposed an algorithm allowing the parallel sensing on multiple sub-bands. However, such approaches always need $L$ sensing times ( $L$ is the number of sub-bands), causing large latency to the spectrum sensing process. Another solution is to employ a wideband circuit [13], [14], which consists of a single RF chain followed by high-speed DSP, to flexibly sense over multiple frequency bands. However, the high-speed DSP is very expensive, power consuming and requires extensive computations. Recent proposals exploit compressed sensing [15], [16] for wideband sensing. It performs sub-Nyquist sampling, thus it can reduce the needs of high-speed data conversion and processing which are required in wideband sensing. 
Unfortunately, the DSA networks must obtain the sparsity as prior knowledge. Moreover, the compressed sensing requires sophisticated hardware architectures [17], [18]. Wideband Orthogonal Frequency Division Multiplexing (OFDM) is anti-multipath interference and flexible in spectrum allocation while the sensing overhead is large.

In[19], the authors try to solve the problem by using analog hardware. The cost of off-the-shelf analog filters and energy detectors is already very low. For example, AD8310, a high performance energy detector, costs only $\$ 4.62$. Costs of low pass filters vary with their filtering bandwidths, but they are usually less than $\$ 5$. Moreover, analog hardware does not have an impact on the total sensing delays. Analog filters have response delays around $n s$ [19].

In this paper, we propose an algorithm based on analog filter for wideband sensing called Exponential and Linear Combined Sensing, which is fast, low-cost and energy-efficient. The basic of our algorithm is that if the sum of energy on a contiguous band is nearly zero, the channels are clear, regardless of the bandwidth. Based on this assume, there is no need to sense the sub-band one by one within the clear channels. Only one measurement is required to probe the energy level of an entire specific bandwidth. We can skip looking into the bandwidth if the observed energy is lower than the given threshold, and assume the channels in this band are clear. To explore the clear channels, we use the exponential strategy, which means that we will extend the scale doubled if the energy in the previous scale is not significant enough. Once there is some energy, we zoom in and then measure the energy at finer granularities. If we find the first channel which is being used, we change into linear strategy, as the name indicates, we scan the next channel in a linear way. Analog filter is chosen as the basic building block for our scheme deliberately. The wideband is divided into various sub-bands by analog filters, and then we use tunable analog filters to adjust the target bandwidth. By using an analog energy detector we can measure the energy of every sub-band.

As it is seen above, Exponential and Linear Combined Sensing(ELCS) builds upon an intelligent algorithm and uses analog hardware as the accompany device. Getting the two strategies combined, the ELCS outperformance compressed sensing for it is quick and easy to implement.

The rest of this paper is organized as follows. Section II describes the spectrum sensing problem and introduces Exponential and Linear Combined Sensing. Section III discusses several additional issues. Section IV offers simulation results and analyzes the computational complexity of the algorithm. We finally make the conclusion in Section IV.

\section{SYSTEM MODEL}

We divide the entire wideband into $L$ sub-bands. That is, we assume the spectrum that we will observe has $L$ channels, each having fixed bandwidth. The bandwidth of each sub-band is fixed but can be different. In this case, a binary hypothesis test can be formulated as follows:

$$
X_{k}=\left\{\begin{array}{r}
N_{k}, H_{0}^{k} \\
S_{k}+N_{k}, H_{1}^{k}
\end{array}\right.
$$

where, $k$ is the $k$-th sub-band. $N$ denotes the noise and $S$ represents the transmitted signal. Thus the hypotheses $H_{0}^{k}$ and $H_{1}^{k}$ means the case that the $k$-th sub-band is clear or is used by a signal respectively. For convenience, we use variable $X_{k} \in\{0,1\}$ to be an indicator that specifies whether sub-band $\boldsymbol{k}$ is occupied or not. To create a spectrum map of the entire spectrum, we need to determine all the values of $X_{k}$.

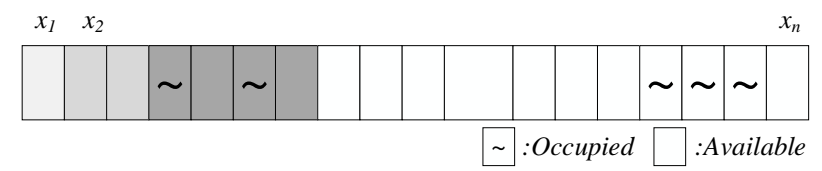

Fig. 1.Wideband spectrum sensing where $x_{i}=1$ if channel $\mathrm{i}$ is occupied, and 0 others

The power detected on sub-band $k$ is denoted as $P_{k}$. The energy detection threshold on sub-band $k$ is $T h_{k}$. We have $X_{k}=0$ if the $P_{k}<T h_{k}, 1$, otherwise. More details about the threshold $T h_{k}$ will be discussed in section IV.

$f_{k}$ is used to denote the starting frequency of the channel $k$. A frequency filter can be positioned over the interval $\left[f_{k}, f_{k+b}\right]$ to separate the signal within the frequency band, where $k$ and $b$ is arbitrarily chosen.

Once we obtain the signal power within the band $P_{[k, k+b-1]}$. Where,

$$
P_{[k, k+b-1]}=P_{k}+P_{k+1}+P_{k+2}+\cdots+P_{k+b-1}
$$

Then set $\mathrm{Th}_{[\mathrm{k}, \mathrm{k}+\mathrm{b}-1]}$ as the corresponding threshold for energy detection. We decide whether the band is clear or not by

$$
\left\{\begin{aligned}
P_{[k, k+b-1]}<T h_{[k, k+b-1]}, & S_{i}=0, i \epsilon k, \ldots, k+b-1 \\
P_{[k, k+b-1]}>T h_{[k, k+b-1]}, S_{i}=1 & \\
& \text { for one or more } i \epsilon\{k, \ldots, k+b-1\}
\end{aligned}\right.
$$

In the second case, we get the information that there is at least one sub-band between channels $[k, k+b-1]$ is occupied.However, we have no idea about how many sub-bands are occupied and where their specific locations in the wideband are.

In general, the wideband is always sparse, which means that the total number of sub-bands being occupied is small and there are plenty of sub-bands that are clear, more specifically, there may be a bunch of channels which are all empty. Thus we can measure not mere one sub-band like sequential scan, instead, a bunch of sub-bands can be detected by only one measurement. The goal for our algorithm is to obtain the spectrum map as fast as possible especially when the wideband is sparse.

\section{A. Basic Algorithm}

Our algorithm is a combination of exponential and linear sensing, similar to [19]. Computational complexity of reconstruction remains the same, linear and 
logarithmic. However, compared with the one in [19], our algorithm is proved to be quicker, more flexible and near optimal for different levels of sparsity.

The algorithm in [19] can reach a good performance when the spectrum is sparse. But as the spectrum utilization becomes higher, it will deteriorate sharply, especially when the spectrum is around half-occupied. The total measurements are even greater than the total number of sub-bands. When the spectrum gets denser, the sensing window expands and shrinks around the same sub-band, spending much time to make sure which sub-band is being used. Avoid moving back and forth to find the occupied sub-bands in its window thus we can decrease the total measurements, in other words, make the sensing quicker. The insight idea of this algorithm is that we use exponential sensing to quickly find out the sequential zeros in the wideband, but unlike [19], we use linear sensing to find the ones in the sensing window step by step, instead of going back and forth around the same frequency. Both strategies are based on the hypothesis that the tunable filter we use can completely separate out the target channels.

In more details, if $P_{[k, k+b-1]}$ is below a threshold, we can deduce that $X_{k}, X_{k+1}, X_{k+2}, \ldots X_{k+b-1}=0$. Otherwise, we need to zoom in for more information about the usage of spectrum in this window. The algorithm starts to find the first band of contiguous zeros. If $X_{k}=0$, then it expands the sensing window, operate the detection to measure $P_{[2,3]}$, the interval in this turn is 2. If $P_{[2,3]}$ is below the threshold, it doubles the interval to $4, P_{[4,7]}$ will be detect in the next measurement. The interval will expand like this if the energy power is small enough, that's why we call it exponential sensing. When $P_{[4,7]}$ is above the threshold, considering the fact that there must be one or more sub-bands in $P_{[4,7]}$, the algorithm changes to linear sense, measuring $X_{4}, X_{5}, X_{6}, X_{7}$ to find out the band occupied. In this way, we can avoid staying around the current sensing space, wasting much time hanging back and forth. Furthermore, it will never need to measure more than the total number of sub-bands like the one in [19], even in the worst case, $O(N)$.

\section{B. Enhanced adaptive algorithm}

Consider this situation in ELCS. When the spectrum is very sparse, maybe 50 channels are occupied in 1000 channels. Due to the randomness of the location of the occupied channels, the interval $d$, which is doubled as long as the power is smaller than then corresponding threshold, can expand to very big. It can reach 32, 64, 128 or even bigger in some extreme cases. Thus when we find the energy power is above the threshold, we have to measure the sub-bands in the interval contiguously, increasing the total measurements greatly. On the other hand, it is not an advantage for us to set the energy threshold when the interval is too big. We will look into the threshold setting in section IV.

Taking all these into consideration, we propose a more intelligent algorithm, which can adaptively adjust the biggest interval according to the sparse of the entire spectrum. Through the previous detection, we can get some information about the sparsity of the spectrum using $k=l_{1} / L$, where $l_{1}$ is the total number of the occupied sub-bands, $L$ is the entire sub-bands in this wideband. A dynamic interval threshold can be set according to the sparsity $k$. At the same time, we use Quicksense as one of the strategies in our enhanced adaptive algorithm for it is well performed when the spectrum is greatly sparse,to be specific, when the sparsity is under $10 \%$. In Quicksense, use the case in basic algorithm as an example, if $P_{[4,7]}$ is above the sum of the energy power, it will shrink its window and measure $P_{[4,5]}$, if $P_{[4,5]}$ still above the threshold respectively, then it continually shrinks to $X_{4}$, if $X_{4}=1$, it then detects $X_{5}$, once $X_{5}=0$, it doubles the interval to 2, measure $P_{[7,8]}$ and so on. This strategy can be used when there are few sub-bands are occupied. As the spectrum becomes denser, we can adjust the biggest interval, the threshold of the window length, dynamically and appropriately based on the sparsity we get from previous detection. In this way, we can further reduce the total measurements and shorten the time of sensing. When the sparsity is over $10 \%$, we change to ELCS, the basic algorithm introduced above. The hybrid adaptive spectrum sensing scheme combines the advantages of the two algorithms, reducing the sensing time effectively.

\section{Channel bonding}

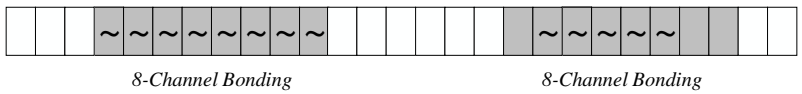

Fig. 2. 8 sub-bands are used as one big channel

It is acknowledged that we will not use only one sub-band in most cases, instead, we often occupy several sub-bands consecutively to satisfy the demand in reality, that is, we may use channel bonding. If we need to use one channel, the sub-bands are grouped and used as a single channel, e.g., group every 8 sub-bands which are consecutively located together. When one sub-band is used, the sub-bands exactly located along its side will be influenced more or less. We can also avoid using them by channel bonding if the grouped channels are less than 8 in this case. So we can set an appropriate grouped number to optimize the detection according to reality. Our ELCS algorithm becomes significantly efficient in this case, the worst measurement is less than 450 (take 1000 sub-bands as the simulation wideband), even less than the total number of occupied channels in the entire wideband. That is because once we find that the energy in this band is abovethe threshold, we assume that the nearby 8 sub-bands are all occupied and then jump to measure the $9^{\text {th }}$ channel behind it.

\section{DISCUSSION}

\section{A. Energy detection threshold}

The variable bandwidths have different noise power, which will influence the threshold. So it is of great importance to deal with this problem. $P_{[i, j]}$, the energy sensed in channel $[i, j]$ equals $S_{[i, j]}+\Omega_{[i, j]}$, where $S_{[i, j]}$ is the sum of energy and $\Omega_{[i, j]}$ is the background noise. $\Omega_{[i, j]}=\sum_{k=i}^{j} \Omega_{k}$ It is obvious that the larger bandwidth it detects, the greater noise power it will observe. We can get a noise map by measuring the noise 
power in empty channels before the actual sensing operation. The noise map shows the noise power $\Omega_{k}$, $0<k<1$, in every sub-band. There is no need to measure the noise frequently to build the noise map as the noise power changes slowly in most cases. To make the map more accurate, we can change the reading frequency according to the randomness of the noise.

Let $M$ denote the total number of energy readings. $\mathrm{S}_{k}$ is the reading sample from channel $k$ and $S_{k}=x_{k}+\omega_{k}$ where $x_{k}$ and $\omega_{k}$ are complex variables denoting the received signal and the noise. We can formulate the average energy reading at channel $k E_{\left[P_{k}\right]}$ as follows:

$$
\begin{aligned}
& \mathrm{E}\left[P_{k}\right]=\frac{1}{M} \sum_{1}^{M}\left|S_{k}\right|^{2}=\frac{1}{M} \sum_{1}^{M}\left|x_{k}+\omega_{k}\right|^{2}(4) \\
= & \frac{1}{M} \sum_{1}^{M}\left(\left|x_{k}\right|^{2}+\left|\omega_{k}\right|^{2}+x_{k}^{\prime} \omega_{k}+x_{k} \omega_{k}^{\prime}\right)(5)
\end{aligned}
$$

As $M$ is large enough so that $\frac{1}{M} \sum_{1}^{M}\left|\omega_{k}\right|^{2}=\Omega_{k}$. As the two random variables $x_{k}$ and $\omega_{k}$ are mutually independent and $\mathrm{E}[\omega] \approx 0$ in AWGN channel , $\sum x * \omega=E[x] * E[\omega] \approx 0$. So we have

$$
\mathrm{E}\left[P_{k}\right]=\frac{1}{M} \sum_{1}^{M}\left|x_{k}\right|^{2}+\Omega_{j}=\mathrm{S}_{j}+\Omega_{j} .
$$

Respectively, $P_{[i, j]}$ is

$$
P_{[i, j]}=\sum_{n=1}^{j} E\left[P_{i}\right]=S_{[i, j]}+\Omega_{[i, j]} \cdot(7)
$$

$\Omega_{[i, j]}$ will be given by the noise map, so if we subtract it from the energy we detect, we can get the signal power. $\Omega_{[i, j]}$ is used as the threshold, and we assume that the channel is occupied when $P_{[i, j]}>\Omega_{[i, j]}$, or else, to be empty. Since the threshold is tightly related to the noise power, it is of great importance to $M$ which has great influence on the accuracy of detection.

\section{B. False Alarm detection or Miss Alarm detection}

As it is discussed above, ELCS is based on energy detection. Unavoidably, the False Alarm and Miss Alarm have to be trade-off by the energy detection threshold. Obviously, Miss Alarm is more harmful compared with False alarm as it may interface the primary user and attempt to use a sub-band which is being used. So we prefer to set the threshold conservatively to minimize the possibility of Miss Alarm. Furthermore, Miss Detection can happen if the primary signal is too weak to be detected out. So it is preferable to sense the narrow band again right before the transmission to make sure the target channel is truly empty.

\section{Performance of analog devices}

In this novel algorithm, we use analog filters and energy detectors which are cheap and energy-efficient. Still, the response delay of these devices is very small, of around hundreds ns. However, an analog filter cannot remove out of band frequencies completely. The residual energy from adjacent channels can increase the overall energy more or less, which may lead to the result of False Alarm. Fortunately, we can use the noise map to calibrate the energy threshold so that the influence of residual energy can be minimized.

\section{SIMULATION RESULTS}

In this section, we illustrate the performance of our spectrum sensing algorithm by computational simulation. Here, at each run, the sub-band which is occupied is randomly chosen by computer program, and the sparse $K$ is varied from 0 to 1 . At each sparse, the simulation is repeated 100 times.

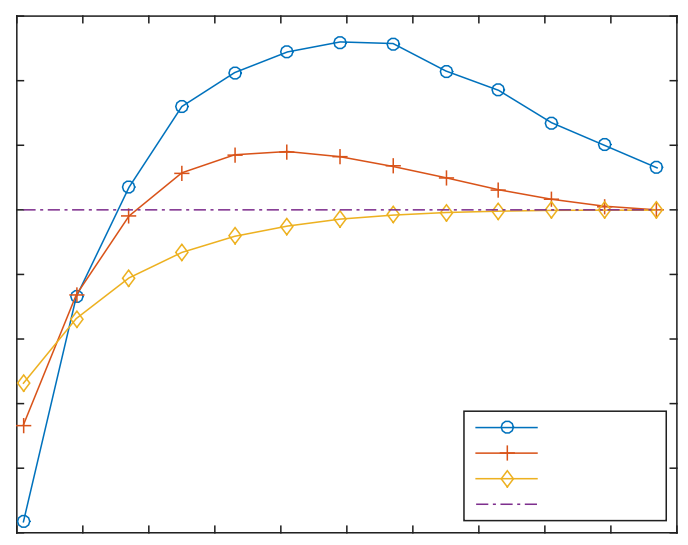

Fig.3.Performance of Quicksense, Quicksense with window threshold (Quicksense-th), ELCS and Sequential scan

The result is showed in figure 3 . The sequential scan always needs 1000 measurements scanning through all the sub-bands. The Quicksense performs well when the wideband is extremely sparse, but it soon becomes bad as the spectrum growing denser. Considering that the back and forth makes the Quicksense deteriorates so fast, we try to set a threshold for the windows scale, when it is long enough and has a big chance to shrink its window in the next turn, we automatically reset the interval to 1 to avoid moving around the same frequency. The orange line Quicksense-th shows that it outperforms the previous algorithm when the sparse is over $20 \%$, but still, both of them are over the total number of sub-band, the Quick sense is even 1.4 times more than L. ELCS is the best one among these four algorithms. When the spectrum is sparse ( $<30 \%$ ), it almost saves 20\% 50\% measurements compared with sequential scan, not to mention the Quicksense. The key insight of it is that when the sum of energy is over the threshold we set, it then detects the sub-band step by step rather than shrinking its window by half, avoiding moving back and forth around the same frequency. It is better to scan the whole window once we find that there are occupied channels in it. 


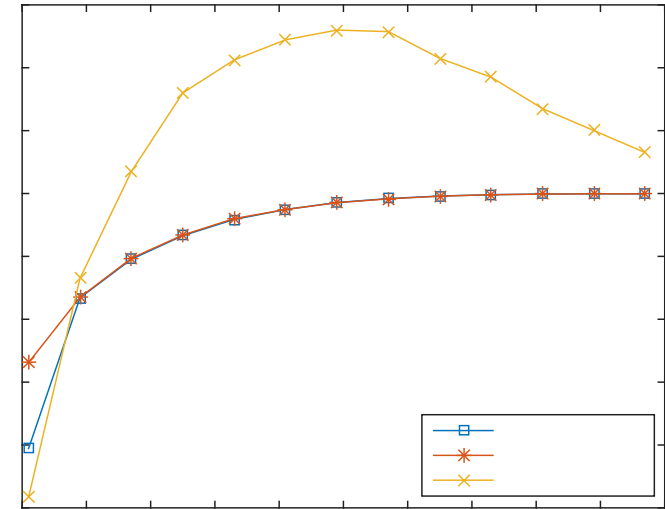

Fig.4.Performance of En-Dynamic ELCS, ELCS and Quicksense

In order to improve the performance of ELCS when the spectrum is extremely sparse, we combine Quicksense with ELCS and get an enhanced dynamic adaptive algorithm. When the spectrum is very sparse, there are rare 1's, 0's dominate the spectrum. It is wise to take advantage of Quicksense in this condition. The small turning point in figure 4 is caused by the switch of these two algorithms.

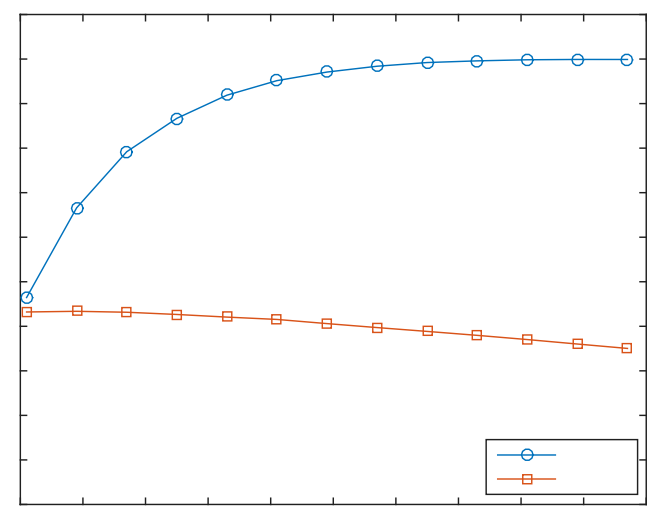

Fig. 5.Performance of ELCS in channel bouding compared with not bouding

Figure5 shows what will happen when we group 8 sub-bands as one channel to use. The other channel which is empty is not bounded. That means if one big channel is used, the consecutively located 8 sub-bands are used. Once we find there is a primary user existing, we can skip 8 sub-bands to detect the next one. In this case, ELCS shows significantly better performance. As the spectrum becomes denser, the total measurements are even less.

\section{CONCLUSION}

In this paper, we propose a novel and fast algorithm combining linear and exponential strategies for the detection of wideband. We have considered the extension of traditional analog filter and energy detector. The realization is simple while the device is cheap. We show that the ELCS can greatly improve the detection of wideband signal compared to sequential scan and
Quicksense. We prove that its complexity is asymptotically same as that of compressed sensing.

\section{REFERENCES}

[1] FCC, "Second memorandum opinion \& order - unlicensed operationin the TV broadcast bands,"FCC 10-174, Sept. 2010

[2] Whitehouse, "President Obama details plan to win the future through expanded wireless access,” 2011.

[3] ECC, "Technical and operational requirements for the possible operation of cognitive radio systems in the 'white spaces' of the frequency band 470-790 MHz,’ECC Report 159, Jan.2011.

[4] D.Cabric, S.M.Mishra, R.W.Brodersen, "Implementation issues in spectrum sensing for cognitive radio,” Proc. of 38th Asilomar conf. on Signals, Systems and Computers, Nov.2004, pp.772-776.

[5] T.Yücek, H.Arslan, "A survey of spectrum sensing algorithms forcognitive radio applications,’IEEE Commun. Surveys and Tutorials,vol.11, No.1, 2009, pp.116-130.

[6] "General survey of radio frequency bands $-30 \mathrm{MHz}$ to $3 \mathrm{GHz}$."

[7] T.Yucek and H.Arslan, "A survey of spectrum sensing algorithms for cognitive radio applications,"Communications Surveys Tutorials, IEEE,2009.

[8] Y.Hur,J.Park, W.Woo, K.Lim, C.-H.Lee, H.S.Kim, J.Laskar, “A wideband analog multi-resolution spectrum sensing (MRSS) technique for cognitive radio (CR) systems," Proc. of IEEE Int Symp. on Circuits and Systems, Kos, Greece, May 2006, pp.4090-4093.

[9] Y.Hur,J.Park, K.Kim, J.Lee, K.Lim, C.-H.Lee, H.S.Kim, J.Laskar,“A cognitive radio (CR) testbed system employing a wideband multi-resolution spectrum sensing (MRSS) technique,”Proc. of 64th IEEE Vehic. Tech. Conf., 2006. Sept. 2006, pp.1-5

[10] L.

DeVito,D.D.Napolitano,S.Rapuano,M.Villanacci, "Eigenvalue-based signal detector for an automatic modulation classifier,”Proc. of IEEE Int. Instrum. and Meas. Tech. Conf., Austin, US, May 2010, pp.1131-1136.

[11] N.Björsell, L.De Vito, S.Rapuano, “A GNU Radio-based signal detector for cognitive radio systems,”Proc. of IEEE Int. Instrum. and Meas. Tech. Conf., Hangzhou, P.R.C., May 2011, pp.1045-1049.

[12] N.M.Neihart, S.Roy, D.J.Allstot, “A parallel, multi-resolution sensing technique for multiple antenna cognitive radios,” Proc. of Int. Symp. on Circuits and Systems, May 2007, pp.2530-2533.

[13] Z.Tian, G.B.Giannakis, "Compressed sensing for wideband cognitive radios," Proc. of IEEE Int. Conf. on Acoustics, Speech and Signal Processing, Honolulu, US, April 2007, vol.IV, pp. 1357-1360.

[14] Z.Quan, S.Cui,H.V.Poor, A.H.Sayed, "Collaborative wideband sensing for cognitive radios,” IEEE Signal Processing Magazine, Nov. 2008, pp.60-73.

[15] J.N.Laska,W.F.Bradley, T.W.Rondeau, K.E.Nolan, and B.Vigoda,"Compressive sensing for dynamic spectrum access networks: Techniques and tradeoffs,”in Proceedings of DySpan, 2011.

[16] M.Rashidi, K.Haghighi, A.Panahi, and M.Viberg, "A NLLS based sub-Nyquist rate spectrum sensing for wideband cognitive radio," in Proceedings of DySpan, 2011.

[17] J.Tropp,M.Wakin,M.Duarte, D.Baron, and R.Baraniuk, "Random filters for compressive sampling and reconstruction,” in Proceedings of ICASSP, 2006.

[18] W.U.Bajwa, J.D.Haupt, G.M.Raz, S.J.Wright, and R.D.Nowak,“Toeplitz-structured compressed sensing matrices,”in Proceedings of SSP, 2007.

[19] S.Yoon,L.E.li,S.C.Liew,R.R.Choudhury,I.Rhee,

K.Tan,"QuickSense:Fast and energy-efficient channel sensing for dynamicspectrum access networks,"INFOCOM, 2013 Proceedings IEEE,pp.2247-2255. 